\title{
The Design of New Geneva Mechanism Contours
}

\author{
Tongyang Hao, Yingjun Hu \\ School of Mechanical Engineering , Tianjin University of Science and Technology , China \\ 1297490976@qq.com
}

Keywords: flexible impact; combination contours; geneva mechanism; ADAMS dynamic simulation

\begin{abstract}
There is flexible impact during the driving of straight geneva mechanism. Therefore, geneva mechanism with combined contours is proposed. The design method of cam contour is adopted in new geneva mechanism (back seeking cam contours according to follower motion). And ADAMS is adopted to carry out the dynamics simulation of the improved geneva mechanism. The experimental result shows that new geneva mechanism reduces the flexible impact, which exists at the moment of round pin passing in and out of the geneva mechanism, and reduces the angular acceleration during the moving of geneva mechanism.
\end{abstract}

\section{Introduction}

Geneva mechanism is simple with small physical dimension and has high mechanical efficiency, which could make steady and intermittent indexing[1]. However, because of the flexible impact that exists in the process of driving motion, geneva mechanism is often used in low speed situation. So reducing the impact and vibration of geneva mechanism becomes the key to improve dynamic performance of geneva mechanism. Fenton[2] proposed to connect two geneva mechanisms in series to eliminate the impact of traditional geneva mechanism, which increased the size of the mechanism itself. Figliolini and Angeles [3] researched the force connection of Geneva mechanism with curved slots. Lee[4] and Huang[5] adopted the design method of cam contour to produce the contour line of geneva mechanism, reducing the impact by changing the geometrical shape of Geneva mechanism. Theoretically, curved slots could eliminate flexible impact. But when round pin reverses, geneva mechanism is incapable of running because of the oversized pressure angle. While straight geneva mechanism has good motion characteristics during the reversing of round pin.

Combining with advantages of curved and straight geneva mechanisms, the paper adopted the method of combined contours to design geneva mechanism. Combined contours mean to adopt curves when round pin is passing in and out of the geneva mechanism and to adopt straight lines when round pin is reversing. Thus, it reduces the impact produced by the passing in and out of round pin and it can make the geneva mechanism run properly. The theoretical research significance of geneva mechanism with combined contours is verified by the simulation result of dynamics. 


\section{The design of combined contours of geneva mechanism}

\subsection{Motion characteristic of normal outside geneva mechanism}

By the mathematical model[2] of traditional geneva mechanism, the mathematical relationship of $\beta$ (angle of rotation) and $\alpha$ (angle of drive plate) is:

$$
\beta=\arctan \frac{b \sin \alpha}{a-b \cos \alpha}
$$

$\mathrm{a}$ is the center distance. $\mathrm{b}$ is the radius of drive plate.

Equation (1) comes at $\omega_{2}$ (angular velocity) and $\varepsilon_{2}$ (angular acceleration).

$$
\begin{aligned}
& \omega_{2}=\frac{d \beta}{d t}=\frac{b(a \cos \alpha-b)}{a^{2}+b^{2}-2 a b \cos \alpha} \omega_{1} \\
& \varepsilon_{2}=\frac{d \beta^{2}}{d^{2} t}=\frac{a b\left(a^{2}-b^{2}\right) \sin \alpha}{\left(a^{2}+b^{2}-2 a b \cos \alpha\right)^{2}} \omega_{1}^{2}
\end{aligned}
$$

\subsection{The choice of follower motion}

The contour design of curved geneva mechanism adopted the design method of cam contour to make a comparison, according to follower motion which included uniform motion, parabolic motion, cosine acceleration, sine acceleration and quintic polynomial.

Table 1 Comparison of motion law

\begin{tabular}{|c|c|c|}
\hline Motion law & Maximum speed & Maximum acceleration \\
\hline Uniform motion & 1.00 & $\infty$ \\
\hline Parabolic motion & 2.00 & 4.00 \\
\hline Cosine acceleration & 1.57 & 4.93 \\
\hline Sine acceleration & 2.00 & 6.28 \\
\hline Quintic polynomial & 1.88 & 5.77 \\
\hline
\end{tabular}

Comparing the motion laws, it shows that polynomial motion law could effectively reduce the velocity and acceleration. Thus the impact and vibration of the mechanism can be avoided.

\subsection{Design conditions}

The design method of cam contours is adopted in the design of curved section. The follower motion used septic polynomial (applied to high velocity and medium load). The theoretical contour line and practical contour line of curved section can be found by reverse method. While straight section can be calculated by traditional design method of geneva mechanism. The intersection of a curve and a straight line is based on the actual demand of work.

The contour lines of combined contours are symmetrical curves. To reduce flexible impact while the round pin is passing in and out, the boundary conditions are:

(1)At the moment of the round pin passing in and out of the rabbet, angular velocity and angular acceleration are both zero.

(2)The curves of angular velocity and angular acceleration are continuous and smooth at the joint of the straight line and curve of geneva mechanism.

\subsection{Mathematical model of motion law of geneva mechanism}

According to the given boundary conditions, combined with formula (1) (3), mathematical expressions of boundary conditions can be calculated.

$$
\begin{gathered}
\left.\beta\right|_{\alpha=-\alpha_{0}}=-\beta_{0} \\
\left.\frac{d \beta}{d \alpha}\right|_{\alpha=-\alpha_{0}}=0
\end{gathered}
$$




$$
\begin{aligned}
& \left.\frac{d^{2} \beta}{d \alpha^{2}}\right|_{\alpha=-\alpha_{0}}=0 \\
& \left.\beta\right|_{\alpha=-\alpha_{p}}=\arctan \frac{-\lambda \sin \alpha_{p}}{1-\lambda \cos \alpha_{p}} \\
& \left.\frac{d \beta}{d \alpha}\right|_{\alpha=-\alpha_{p}}=\frac{-\lambda\left(\cos \alpha_{p}-\lambda\right)}{\rho^{\prime}} \\
& \left.\frac{d^{2} \beta}{d \alpha^{2}}\right|_{\alpha=-\alpha_{p}}=\frac{-\lambda\left(\lambda^{2}-1\right) \sin \alpha_{p}}{\rho^{\prime 2}} \\
& \left.\frac{d^{3} \beta}{d \alpha^{3}}\right|_{\alpha=-\alpha_{p}}=-\lambda\left(\lambda^{2}-1\right) \frac{\rho^{\prime} \cos \alpha_{p}-4 \lambda \sin ^{2} \alpha_{p}}{\rho^{\prime 3}} \\
& \left.\frac{d^{4} \beta}{d \alpha^{4}}\right|_{\alpha=-\alpha_{p}}=\frac{\left(\rho^{\prime 2}+4 \lambda \cos \alpha_{p} \rho^{\prime}+8 \lambda \rho^{\prime}-24 \lambda^{2} \sin ^{2} \alpha_{p}\right)}{\rho^{\prime 6}} \lambda\left(\lambda^{2}-1\right) \sin \alpha_{p} \rho^{\prime 2} \\
& \lambda=b / a ; \rho^{\prime}=1+\lambda^{2}-2 \lambda \cos \alpha_{p}
\end{aligned}
$$

The design of curved section adopted septic polynomial. The rotor angle of reticule is:

$$
\beta=C_{0}+C_{1}\left(\alpha+\alpha_{0}\right)+C_{2}\left(\alpha+\alpha_{0}\right)^{2}+C_{3}\left(\alpha+\alpha_{0}\right)^{3}+C_{4}\left(\alpha+\alpha_{0}\right)^{4}+C_{5}\left(\alpha+\alpha_{0}\right)^{5}+C_{6}\left(\alpha+\alpha_{0}\right)^{6}+C_{7}\left(\alpha+\alpha_{0}\right)^{7}
$$

$C_{0} \backsim C_{7}$ are undetermined coefficients.

Put $(4) \sim(6)$ into (12):

$$
C_{0}=-\beta_{0} ; C_{1}=0 ; C_{2}=0 ;
$$

Put the coefficients into (12). A new expression of rotor angle of reticule can be found:

$\beta=-\beta_{0}+C_{3}\left(\alpha+\alpha_{0}\right)^{3}+C_{4}\left(\alpha+\alpha_{0}\right)^{4}+C_{5}\left(\alpha+\alpha_{0}\right)^{5}+C_{6}\left(\alpha+\alpha_{0}\right)^{6}+C_{7}\left(\alpha+\alpha_{0}\right)^{7}$

Put (7) (11) into (13). Then homogeneous linear equations (14) can be found. According to practical installation dimensions and drive ratio, parameters $a, b 、 \alpha_{0} 、 \beta_{0}$ and $\alpha_{p}$ can be found. Then put these parameters into (14), using MATLAB to come at the solution of the system of homogeneous equations.

$$
\left\{\begin{array}{l}
C_{3}\left(\alpha_{0}-\alpha_{p}\right)^{3}+C_{4}\left(\alpha_{0}-\alpha_{p}\right)^{4}+C_{5}\left(\alpha_{0}-\alpha_{p}\right)^{5}+C_{6}\left(\alpha_{0}-\alpha_{p}\right)^{6}+C_{7}\left(\alpha_{0}-\alpha_{p}\right)^{7}=\arctan \frac{-\lambda \sin \alpha_{p}}{1-\lambda \cos \alpha_{p}}+\beta_{0} \\
3 C_{3}\left(\alpha_{0}-\alpha_{p}\right)^{2}+4 C_{4}\left(\alpha_{0}-\alpha_{p}\right)^{3}+5 C_{5}\left(\alpha_{0}-\alpha_{p}\right)^{4}+6 C_{6}\left(\alpha_{0}-\alpha_{p}\right)^{5}+7 C_{7}\left(\alpha_{0}-\alpha_{p}\right)^{6}=\frac{\lambda\left(\cos \alpha_{p}-\lambda\right)}{\rho^{\prime}} \\
6 C_{3}\left(\alpha_{0}-\alpha_{p}\right)+12 C_{4}\left(\alpha_{0}-\alpha_{p}\right)^{2}+20 C_{5}\left(\alpha_{0}-\alpha_{p}\right)^{3}+30 C_{6}\left(\alpha_{0}-\alpha_{p}\right)^{4}+42 C_{7}\left(\alpha_{0}-\alpha_{p}\right)^{5}=\frac{-\lambda\left(\lambda^{2}-1\right) \sin \alpha_{p}}{\rho^{\prime 2}} \\
6 C_{3}+24 C_{4}\left(\alpha_{0}-\alpha_{p}\right)+60 C_{5}\left(\alpha_{0}-\alpha_{p}\right)^{2}+120 C_{6}\left(\alpha_{0}-\alpha_{p}\right)^{3}+210 C_{7}\left(\alpha_{0}-\alpha_{p}\right)^{4}=\frac{\lambda\left(\lambda^{2}-1\right)\left(\rho^{\prime} \cos \alpha_{p}-4 \lambda \sin ^{2} \alpha_{p}\right)}{\rho^{\prime 3}} \\
24 C_{4}+120 C_{5}\left(\alpha_{0}-\alpha_{p}\right)+360 C_{6}\left(\alpha_{0}-\alpha_{p}\right)^{2}+840 C_{7}\left(\alpha_{0}-\alpha_{p}\right)^{3}=\frac{\rho^{\prime 2} \lambda\left(\lambda^{2}-1\right) \sin \alpha\left(\rho^{\prime 2}+4 \lambda \cos \alpha \rho^{\prime}+8 \lambda \rho^{\prime}-24 \lambda^{2} \sin ^{2} \alpha\right)}{\rho^{\prime 6}}
\end{array}\right.
$$

When the expression of motion of geneva mechanism is confirmed, the polar equation of theoretical contour line can be calculated by reverse method.

$$
\left\{\begin{array}{l}
\rho=\sqrt{a^{2}+b^{2}-2 a b \cos \alpha} \\
\theta=\beta-\arctan \frac{b \sin \alpha}{a-b \cos \alpha}
\end{array}\right.
$$

According to the equation of theoretical contour line, the polar equation of practical contour line can be calculated. 


$$
\left\{\begin{array}{l}
\rho_{p}=\sqrt{\rho^{2}+r^{2}-2 \rho r \cos \gamma} \\
\theta_{p}=\theta-\arctan \frac{r \sin \gamma}{\rho-r \cos \gamma}
\end{array}\right.
$$

$\gamma=\arctan \frac{\dot{\rho}}{\rho \dot{\theta}}(\gamma$ is the pressure angle of the theoretical contour line of curved section $), \mathrm{r}$ is the radius of the round pin.

\subsection{Design example}

According to the production requirement of a packinghouse, new geneva mechanism with single round pin and 10 positions is designed. Specific parameters are:

$$
a=300 \mathrm{~mm} ; b=92 \mathrm{~mm} ; r=12 \mathrm{~mm} ; \alpha_{0}=72^{\circ} ; \beta_{0}=18^{\circ} ; \alpha_{p}=12^{\circ}
$$

Put those parameters into formula (13). Making use of MATLAB, the solution of system of linear equations can be calculated:

$$
C_{3}=0.7828 ; C_{4}=-1.2012 ; C_{5}=0.9932 ; C_{6}=-0.4397 ; C_{7}=0.0784 ;
$$

Put undetermined coefficient into formula (12):

$$
\beta=-\frac{\pi}{10}+0.7828\left(\alpha+\alpha_{0}\right)^{3}-1.2012\left(\alpha+\alpha_{0}\right)^{4}+0.9932\left(\alpha+\alpha_{0}\right)^{5}-0.4397\left(\alpha+\alpha_{0}\right)^{6}+0.0784\left(\alpha+\alpha_{0}\right)^{7}
$$

Put the formula into (15) (16) to acquire part of the theoretical contour line and practical contour line. The curved part of theoretical and practical contour line is shown in Fig.1 and Fig.2.

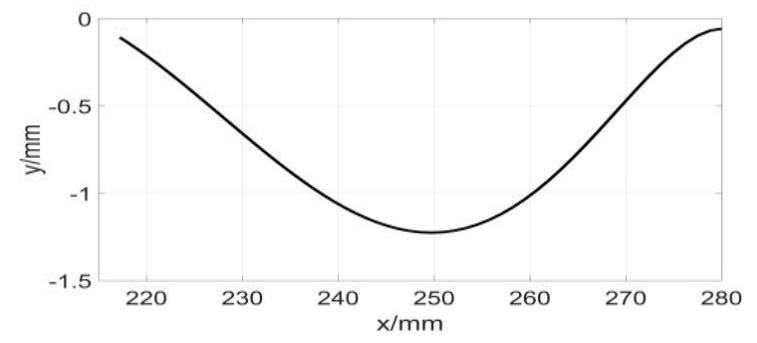

Fig 1. Theoretical contour line

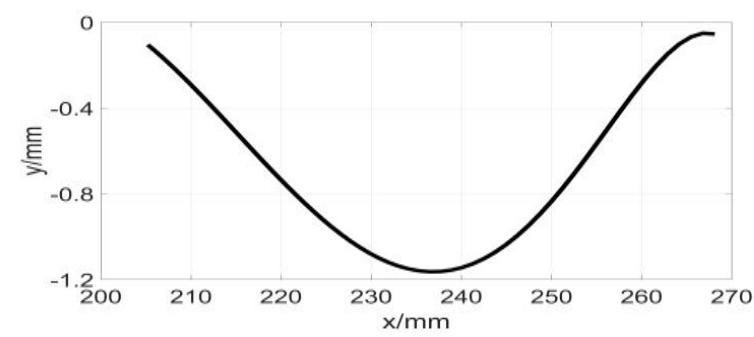

Fig 2. Practical contour line

\section{Single-edge contact dynamic model simulation with clearance}

During practical driving, clearance between round pin and geneva mechanism is unavoidable and necessary. Excessive clearance would cause noise and vibration. When the clearance is too small, it could cause impact and interference between round pin and geneva mechanism. So a dynamic model considering with clearance is more persuasive. At present, most researches about high-velocity geneva mechanism analyzed kinematics. Few documents analyzed geneva mechanism with curved and straight contours considering with clearance. The paper built a single-edge contact dynamic model with clearance $(e=0.1 \mathrm{~mm})$, making the passing in and out of round pin as a period. It has practical significance on verifying that new geneva mechanism can reduce flexible impact by the simulation result.

According to the practical need of works, set parameters in ADAMS clutch plate speed $\omega_{1}=30 \mathrm{r} / \mathrm{min}$, stiffness coefficient of clutch and geneva material $K=1 \times 10^{8} \mathrm{~N} / \mathrm{m}$, poisson ratio $\mu=0.29$, elasticity modulus $E=207 G P a$, nonlinear index of force $e=2.2$ (applied for steel), maximal damping coefficient $C_{\max }=1 \times 10^{4} \mathrm{~N} \cdot \mathrm{s} / \mathrm{m}$, maximal contact depth of damping coefficient $\sigma_{\max }=1 \times 10^{-4} \mathrm{~m}$. There are normal contact force and tangential friction force when round pin and geneva mechanism contacts. Parameters of coulomb friction need to be defined. Static friction coefficient is 0.3 . Dynamic friction coefficient is 0.1 . Relative sliding velocity corresponding maximal static friction is $0.1 \mathrm{~m} / \mathrm{s}$. Sliding velocity corresponding dynamic friction is $1 \mathrm{~m} / \mathrm{s}$. Simulation time is $1 \mathrm{~s}$. Simulation step number is 1000 . 


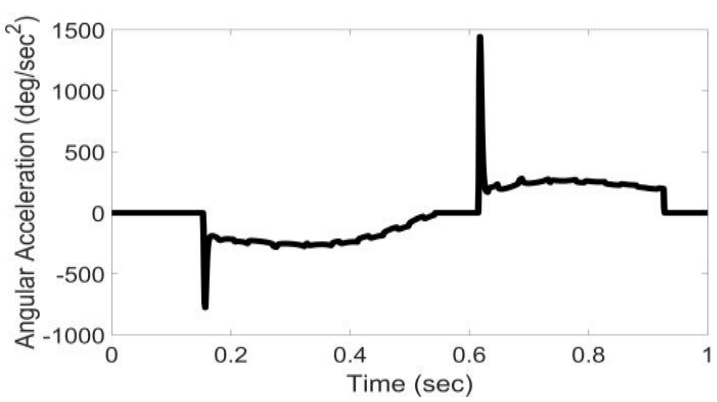

Fig.3. Angular acceleration of traditional geneva mechanism

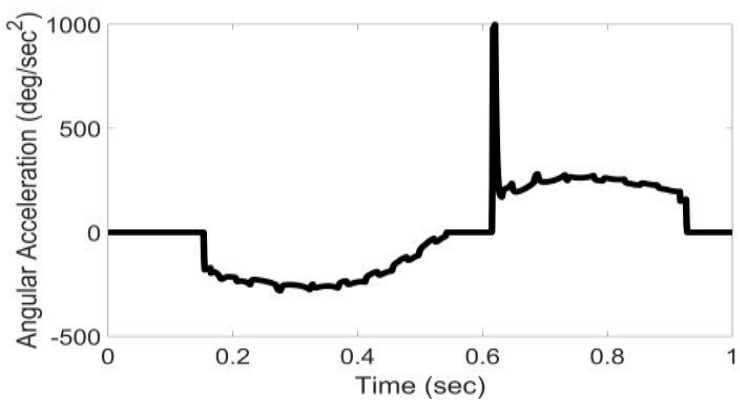

Fig.4. Angular acceleration of new geneva mechanism

The changing curve of angular acceleration of traditional geneva mechanism is as Fig.3. When the round pin passed in and out of radial slot, the curve has an obvious bounce. At the moment, flexible impact appears. While the round pin is passing geneva mechanism horizontally $\left(\alpha=0^{\circ}\right)$, angular acceleration is zero and angular velocity maintains uniform. Then it will have reverse impact. Considering poor lubrication of geneva mechanism and the impact of working accuracy, there will be slight vibration in the whole motor process.

As in figure4, compared with traditional geneva mechanism, new geneva mechanism reduces flexible impact, reverse impact and peak angular acceleration. The angular accelerations of round pin passing in and out of the geneva mechanism are respectively as $\varepsilon_{a}$ and $\varepsilon_{b}$. Reversing angular acceleration is $\varepsilon_{c}$. Peak angular acceleration is $\varepsilon_{d}$. The simulation data is in table 2 .

Table 2 Simulation data comparison of angular acceleration of geneva mechanism

\begin{tabular}{|c|c|c|c|c|}
\hline name & $\varepsilon_{a}\left({ }^{o} / s^{2}\right)$ & $\varepsilon_{b}\left({ }^{o} / s^{2}\right)$ & $\varepsilon_{c}\left({ }^{o} / s^{2}\right)$ & $\varepsilon_{d}\left({ }^{o} / s^{2}\right)$ \\
\hline Traditional geneva mechanism & -776 & 202 & 1440 & 251 \\
\hline new geneva mechanism & -196 & 159 & 990 & 238 \\
\hline
\end{tabular}

\section{Conclusion}

The paper adopted and elaborated systematically the contour line design method of geneva mechanism with straight and curved contours. The boundary conditions of reducing impact and vibration are defined. And the mathematical model of follower motion and contour line is established. Taking the intelligent foreign body removal mechanism as the research background, dynamic simulation of high-velocity geneva mechanism has been made. The simulation result illustrates that the design of new geneva mechanism can effectively reduce the impact and vibration.

\section{References}

[1] R.G. Fenton, Y. Zhang, J. Xu, Development of a new Geneva mechanism with improved kinematic characteristics, ASME Journal of Mechanical Design 113 (1991) 40 - 45.

[2] Y. Zhang, R.G. Fenton, J. Xu, Two station Geneva mechanisms, ASME Journal of Mechanical Design 116 (1994) $647-653$.

[3] G. Figliolini, J. Angeles, Synthesis of conjugate Geneva mechanisms with curved slots, Mechanism and Machine Theory 37 (2002)1043 - 1061.

[4] H.P. Lee, Design of a Geneva mechanism with curved slots using parametric polynomials, Mechanism and Machine Theory 33 (3)(1998) 321 - 329.

[5] J.J. Lee, K.F. Huang, Geometry analysis and optimal design of Geneva mechanisms with curved slots, Journal of Mechanical Engineering Science, Proceedings of the Institution of Mechanical Engineers, Part C 218 (4) (2004) 449 - 459. 